Check for updates

Cite this: Phys. Chem. Chem. Phys., 2019, 21, 18119

Received 24th May 2019 , Accepted 2nd August 2019

DOI: 10.1039/c9cp02950h

rsc.li/pccp

\section{Ultrafast ring-opening and solvent-dependent product relaxation of photochromic spironaphthopyran $\dagger$}

\author{
Simon F. Bittmann, (D) $\ddagger^{a}$ Raison Dsouza, (D) $\ddagger^{\text {ab }}$ Khalid M. Siddiqui, $\ddagger^{a}$ Stuart A. Hayes, ${ }^{a}$ \\ Andreas Rossos, ${ }^{a}$ Gastón Corthey, (iD ac Michał Kochman, iD ad \\ Valentyn I. Prokhorenko, ${ }^{a}$ R. Scott Murphy, (D) e Heinrich Schwoerer (D) a and \\ R. J. Dwayne Miller (iD *af
}

\begin{abstract}
The ultrafast dynamics of unsubstituted spironaphthopyran (SNP) were investigated using femtosecond transient UV and visible absorption spectroscopy in three different solvents and by semi-classical nuclear dynamics simulations. The primary ring-opening of the pyran unit was found to occur in 300 fs yielding a non-planar intermediate in the first singlet excited state $\left(\mathrm{S}_{1}\right)$. Subsequent planarisation and relaxation to the product ground state proceed through barrier crossing on the $S_{1}$ potential energy surface (PES) and take place within 1.1 ps after excitation. Simulations show that more than $90 \%$ of the trajectories involving $\mathrm{C}-\mathrm{O}$ bond elongation lead to the planar, open-ring product, while relaxation back to the $\mathrm{S}_{0}$ of the closed-ring form is accompanied by $\mathrm{C}-\mathrm{N}$ elongation. All ensuing spectral dynamics are ascribed to vibrational relaxation and thermalisation of the product with a time constant of $13 \mathrm{ps}$. The latter shows dependency on characteristics of the solvent with solvent relaxation kinetics playing a role.
\end{abstract}

\section{Introduction}

Photochromic compounds exhibit dramatic changes in their structural, chemical and physical properties upon absorption of light which are generally reversible. ${ }^{1}$ This makes them suitable for promising applications, for instance as photoswitches, ${ }^{2-4}$ memory devices $^{5,6}$ and as tools in biological imaging, ${ }^{7}$ motivating research on their synthesis and characterisation. ${ }^{8,9}$ An important class of photochromics is that of spiropyrans, ${ }^{10}$ which are composed of nearly orthogonal indoline and pyran subunits related by a common $\mathrm{sp}^{3}$-hybridised spiro-carbon atom, but can differ in their substituents (see Fig. 1). A common view of the photophysical

${ }^{a}$ Max Planck Institute for the Structure and Dynamics of Matter, Center for Free Electron Laser Science, Luruper Chaussee 149, 22761, Hamburg, Germany. E-mail: dwayne.miller@mpsd.mpg.de

${ }^{b}$ Department of Physics, University of Hamburg, Jungiusstrasse 9, 20355 Hamburg, Germany

${ }^{c}$ Instituto de Nanosistemas, Universidad Nacional de San Martin, San Martin, Buenos Aires, Argentina

${ }^{d}$ Department of Physics, Chemistry and Biology (IFM), Linköping University, Linköping, Sweden

${ }^{e}$ Department of Chemistry and Biochemistry, Research and Innovation Centre, University of Regina, 3737 Wascana Parkway, Regina, SK S4S OA2, Canada

${ }^{f}$ Departments of Chemistry and Physics, University of Toronto, 80 St. George Street, Toronto, ON M5S3H6, Canada

$\dagger$ Electronic supplementary information (ESI) available. See DOI: 10.1039/c9cp02950h \# S. F. Bittmann, R. Dsouza and K. M. Siddiqui contributed equally to this work. properties of spiropyrans is that the two subunits are electronically uncoupled in the closed (spiro-) form and thus, its absorption spectrum lies in the ultraviolet (UV) region of the electromagnetic spectrum $(\lambda<400 \mathrm{~nm})$. When a UV photon is absorbed by the molecule, the bond between the spiro-carbon and the oxygen in the pyran ring undergoes cleavage leading to a cascade of processes, including opening of the pyran ring,

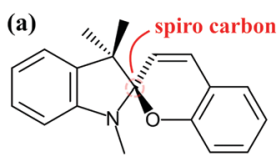

BIPS: spiro form

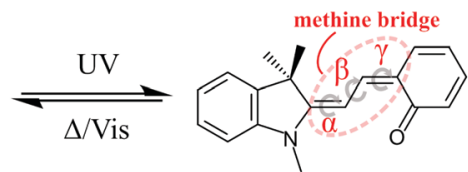

BIPS: merocyanine form (MC)

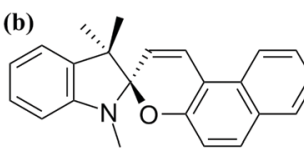

SNP: spiro form
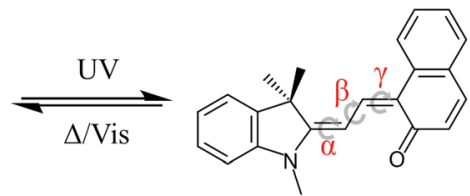

SNP: MC form
Fig. 1 Photochemical reaction scheme of spiropyran conversion to merocyanine shown for indolinobenzospiropyran (BIPS, a) and indolinonaphthospiropyran (SNP, b). Due to rotation about the three bonds in the methine bridge (dihedral angles $\alpha, \beta$ and $\gamma$ ), up to 8 isomers are possible out of which the trans-trans-cis (TTC) configuration is shown. The reverse reaction can be achieved thermally or triggered with visible light. 
hybridisation of the spiro-carbon and conformational isomerisation. These structural changes yield a planar isomer called merocyanine (MC), in which the two moieties are linked by a methine bridge and become electronically coupled. The improved wave function overlap and delocalisation causes a reduction of the energy difference between the highest occupied and the lowest unoccupied molecular orbital giving rise to an intense absorption of the $\mathrm{MC}$ in the visible range. Consequently, the MC form appears coloured in solution. Eight different conformers are in principle possible due to an additional degree of freedom brought upon by the formation of the methine bridge. In Fig. 1, one such conformer is shown as an example. Which conformers are most stable depends on the respective spiropyran derivative ${ }^{11}$ and the solvent environment. ${ }^{12}$

Understanding the fundamental photophysical processes of spiropyrans has been a matter of great interest for the photochemistry community. In 1952, Fischer and Hirshberg were the first to synthesise spiropyrans and also to characterise the role of temperature and the wavelength of light on the behaviour of this class of compounds. ${ }^{13}$ Since then, several studies have been carried out investigating different aspects, such as structural changes during the reaction, their time scales and the quantum yields of possible MC isomers. ${ }^{14,15}$

The most studied spiropyrans have been indolinobenzospiropyran (BIPS, see Fig. 1a) and its 6-nitro $\left(-\mathrm{NO}_{2}\right)$ substituted analogues. However, the time scale for the ring-opening reaction remains a debated point. In the early 90's, Ernsting and coworkers reported the results of optical pump-probe experiments in the visible range carried out on BIPS solutions in $n$-pentane ${ }^{16,17}$ as well as ethanol ${ }^{18}$ and observed the formation of merocyanine species upon excitation at $308 \mathrm{~nm}$ with a rise time of $\sim 0.9 \mathrm{ps}$. They found that the spectrum in the $450-600 \mathrm{~nm}$ range at $30 \mathrm{ps}$ resembled that of one recorded in the microsecond timescales suggesting that the product is well-established within this time frame. From a sterics argument they predicted the observed species to be either in a trans-trans-cis (TTC) or cis-trans-cis (CTC) conformation or both. A transient mid-infrared study in 2003 from Rini et al. cast doubt on the time scale of this photochemical reaction. ${ }^{19}$ Based on their measurements, they concluded that a rapid $\mathrm{S}_{1} \rightarrow \mathrm{S}_{0}$ internal conversion was the dominant relaxation channel with a quantum yield of $\sim 90 \%$, while the remaining $10 \%$ goes into forming the product via an unknown intermediate with a time constant of $28 \mathrm{ps}$. On the other hand, a water soluble, pyridinium substituted BIPS, which can be expected to exhibit dynamics similar to BIPS, was investigated by Kohl-Landgraf et al. and the MC formation following $350 \mathrm{~nm}$ excitation was found to occur within a picosecond. ${ }^{20}$

Spironaphthopyran (SNP), which is closely related to BIPS (see Fig. 1b), has been reported to display transient photochromism even in the crystalline state. ${ }^{21}$ This renders it a promising target for time-resolved crystallographic studies, which have the capability to unambiguously resolve the question of the MC formation time scale and to provide insight into the structural isomerisation dynamics with atomic resolution, ${ }^{22}$ motivating our interest in this system.
For SNP, a rise time of 1.4 ps for the MC absorption band in solution has been reported. ${ }^{16,17}$ However, detailed transient absorption data with sub-ps resolution has only been measured up to 8 ps after UV excitation and only for the non-polar solvent $n$-pentane. As mentioned, the solvent environment is known to influence the equilibrium between different MC conformers depending on the solvent polarity and capability of forming hydrogen bonds. ${ }^{12}$ What role this plays towards the MC formation dynamics however warrants further investigation. Moreover, studies of unsubstituted spiropyrans have mainly been carried out with excitation directly to the $\mathrm{S}_{1}$ state $(E \sim 4 \mathrm{eV})$. Excitation into higher lying electronic states than $\mathrm{S}_{1}$ can lead to larger excess energies being deposited into the system which can potentially influence the outcome of a photochemical reaction..$^{23-26}$ In this context, the solvent can play a determining role on the time scales of vibrational relaxation, as has been reported for instance in the case of 9-methyladenine. ${ }^{27}$

A number of theoretical studies concerning the ring-opening mechanism of spiropyran have been carried out more recently, treating smaller model systems at a high level of theory (CASPT2/CASSCF). ${ }^{28}$ These studies have provided useful insight into the relevant modes and reaction channels leading to either the closed or the open form, but give no direct information about the respective time scales. The latter can be obtained by carrying out excited state dynamics simulations, which has so far only been reported for BIPS. ${ }^{29}$

In this work, we investigated the photochemical dynamics of spironaphthopyran up to extended time scales (one nanosecond) using $266 \mathrm{~nm}$ femtosecond excitation (4.6 eV) and comparing between a non-polar ( $n$-hexane), a polar aprotic (acetonitrile) and a polar protic (ethanol) solvent environment. We performed femtosecond transient absorption spectroscopy covering the visible $(380-700 \mathrm{~nm})$ as well as the ultraviolet spectral range (250-350 nm), where electronic changes associated with the ground state of the spiro-species can be observed. Furthermore, we carried out semi-classical excited state dynamics simulations treating the complete molecule with time-dependent density functional theory and simulating a sufficient number of trajectories to be able to compare the time scales between simulations and experiments. In addition to gaining insight into the ringopening mechanism and the involved electronic states from theory, this makes it possible to differentiate between chemical reaction dynamics and product relaxation in the analysis of the spectroscopic data.

\section{Methods}

\subsection{Computational methods}

The modified-surface hopping dynamics are calculated using the adiabatic electronic energies and gradients calculated on the fly at time-dependent density functional theory (TDDFT) level of theory using the $a b$ initio electronic structure package Gaussian $09 .{ }^{30}$ We treated the electronic structure of the SNP molecule with the use of density functional theory (DFT) for the ground electronic state in combination with (linear response) 
time-dependent DFT (TDDFT) for the relevant excited states. The reactant geometry of the ground state was optimized using DFT using CAM-B3LYP ${ }^{31}$ functional with a def2SVP ${ }^{32}$ basis set. CAM-B3LYP is a long range-corrected functional, owing to which its accuracy for excited-state potential energy surfaces is superior to conventional hybrid functionals. Subsequently, the first singlet excited state $\left(\mathrm{S}_{1}\right)$ was optimised using the same functional and basis set using TDDFT within Tamn-Damncoff Approximation. These calculations were also performed using Gaussian 09.

The simulations assumed that the vertical excitation was instantaneous, with an initial phase space distribution generated by using a vibrational Wigner distribution for the ground state minimum. The phase space coordinates were generated from the total distribution using Monte Carlo sampling. As a by-product of this sampling, the absorption spectra were also calculated from 500 configurations. A spectral window based on experimental excitation energy was used to pick the initial conditions. A total of 30 trajectories were chosen from a larger randomly selected set on the basis of the computed $S_{0}-S_{2}$ and $S_{0}-S_{3}$ transition probabilities (see ESI $\dagger$ ). These trajectories were propagated for $1.1 \mathrm{ps}$ with time steps of $0.5 \mathrm{fs}$ in singlet excited states $S_{2}$ and $S_{3}$.

\subsection{Experimental details}

1,3,3-Trimethylindolino- $\beta$-naphthopyrylospiran (SNP) powder was purchased from TCI Chemicals, Japan and used without further purification. Spectroscopic grade $n$-hexane, ethanol and acetonitrile were used to prepare SNP solutions with a concentration of $1.5 \mathrm{mM}$. To obtain the respective spectra of merocyanine, the solutions were irradiated continuously with $266 \mathrm{~nm}$ laser light until reaching a photostationary state.

Transient absorption experiments were performed using a titanium:sapphire (Ti:Sa) femtosecond laser system (800 nm central wavelength, $1 \mathrm{kHz}$ ). In all measurements, the samples were excited at $266 \mathrm{~nm}$ with a fluence of $4 \mathrm{~mJ} \mathrm{~cm} \mathrm{~cm}^{-2}$ (490 nJ pulse energy with a beam diameter of $120 \mu \mathrm{m}$ FWHM) and a repetition rate of $500 \mathrm{~Hz}$. The visible probe light $(380-700 \mathrm{~nm})$ was generated by focusing $800 \mathrm{~nm}$ pulses in water $(200 \mu \mathrm{m}$ cuvette), with the magic angle $\left(54.7^{\circ}\right)^{33}$ between the pump and probe polarisations. For the UV supercontinuum $(250-350 \mathrm{~nm})$, $400 \mathrm{~nm}$ pulses passing through a quarter-wave plate to create circular polarised light were focused in a rotating [001] calcium fluoride crystal. ${ }^{34}$ The polarisation of the resulting UV continuum was not modified and assumed to be circular. Sample solutions were flowed through an in-line UV-quartz cuvette with a $200 \mu \mathrm{m}$ thin window. The transient absorption signal was calculated as:

$$
\Delta A(\lambda, \Delta t)=-\log \left(\frac{I_{\mathrm{ON}}(\lambda, \Delta t)}{I_{\mathrm{OFF}}(\lambda)}\right)
$$

where $I_{\mathrm{OFF}}(\lambda)$ and $I_{\mathrm{ON}}(\lambda, \Delta t)$ are the background-corrected probe spectra transmitted through the unexcited and the excited sample, respectively, $\Delta t$ is the time delay between pump and probe and $\lambda$ is the probe wavelength. Keeping the probe repetition rate at $1 \mathrm{kHz}$, the $I_{\mathrm{OFF}}(\lambda)$ and $I_{\mathrm{ON}}(\lambda, \Delta t)$ spectra were recorded alternately. For the UV/UV measurements, every third and fourth probe pulse was blocked using a mechanical chopper to enable the subtraction of the pump scattering background at $266 \mathrm{~nm}$ (see ESI $\dagger$ ). ${ }^{35}$ The temporal resolution was determined from a cross-correlation measurement in the neat solvent as $170 \pm 10 \mathrm{fs}$ and the sensitivity of the measurement was $10^{-4} \mathrm{OD}$.

\section{Results and discussions}

\subsection{Steady-state spectra}

The UV/vis absorption spectra of $1.5 \mathrm{mM}$ solutions of SNP in hexane, ethanol and acetonitrile are shown in Fig. 2. At thermal equilibrium, the molecules exist mainly in the closed form (SNP) which only absorbs below $380 \mathrm{~nm}$. From this spectrum, three main bands can be seen, which show no significant solvent dependence.

The inset of Fig. 2 shows the respective absorption spectra of the merocyanine form in the photostationary state. Immediately obvious are their different shapes and/or positions. The MC spectrum in hexane displays a maximum at $\sim 520 \mathrm{~nm}$ and an additional spectral feature on either side of it. For acetonitrile and ethanol, once more at least three peaks can be deciphered. Two are intense peaks with the longest wavelength peak being the most intense and one smaller shoulder at the lower wavelength tail. The MC spectra in ethanol and acetonitrile have similar shapes and are red-shifted with respect to the corresponding spectrum in hexane. The bathochromic shift is most pronounced for ethanol showing a $17 \mathrm{~nm}$ shift of the lower absorption edge with respect to hexane and $5 \mathrm{~nm}$ with respect to acetonitrile. Notably, the overall absorption under the same excitation conditions follows the same trend with hexane showing the least and ethanol the highest absorption (see the scaling factors in Fig. 2). This hints at the solvent acting to stabilise the merocyanine form, which is discussed further in the ESI. $\dagger$

\subsection{Transient absorption experiments}

3.2.1 UV-visible. Measured transient absorption spectra of SNP dissolved in the three respective solvents are shown in

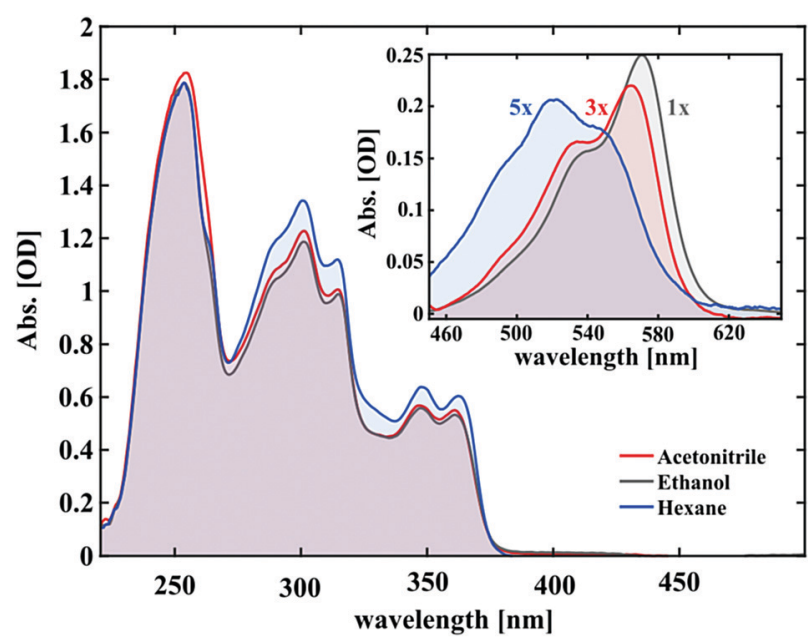

Fig. 2 Steady-state absorption spectra of $1.5 \mathrm{mM} \mathrm{SNP} \mathrm{solutions} \mathrm{in} \mathrm{the} \mathrm{UV}$ and visible range. The inset shows the merocyanine spectra in the steady state under UV irradiation (photostationary state), scaled for comparison. 
Fig. $3 \mathrm{a}-\mathrm{c}$ for the first $14 \mathrm{ps}$ after $266 \mathrm{~nm}$ excitation. Spectral cuts at chosen time delays from scans covering the range up to 100 ps after excitation are presented in the middle panel of the figure. Measurements up to one nanosecond show that there is no significant spectral evolution beyond 100 ps (see ESI $\dagger$ ). The signal observed at time zero is due to the cross-phase modulation (CPM) of the pump and the probe pulse in the solvent. Around $0.3 \mathrm{ps}$, a broad spectrum appears peaking at $450 \mathrm{~nm}$ with an extended tail reaching $650 \mathrm{~nm}$. This signal decays within 1-2 picoseconds accompanied by the emergence of a band in the visible region (450-650 $\mathrm{nm}$ ). Closer inspection of the first two picoseconds reveals that between 0.3 and $1.2 \mathrm{ps}$, the maximum continuously shifts from the initial band to the spectrum centered around $550 \mathrm{~nm}$ (see insets of Fig. 3a-c). From two picoseconds onwards, the initially structureless spectrum evolves over time towards the steady-state spectrum in the respective solvent becoming narrower and more intense.
In the ethanol solution, an additional red-shift in wavelength most prominently within $2-7$ ps can be observed, which is absent in case of the other solvents.

To analyse the data in more detail, we have applied two methods, a global fit routine ${ }^{36}$ on the one hand and a dynamic Gaussian line shape analysis on the other hand. The decayassociated spectra (DAS) from global fitting using four exponential components for the time window between 0.3 and $14 \mathrm{ps}$ are shown in the lowest panel of Fig. 3. A single component with a lifetime of $\sim 1.0$ ps (DAS2, blue line) describes both the decay of the initially broad spectrum (positive sign of DAS2) and the rise of the band between 450 and $600 \mathrm{~nm}$ (negative sign of DAS2) in all solvents. The decay-associated spectrum with the shortest lifetime of around 0.3 ps (DAS1, orange line) has a positive amplitude between 450 and $600 \mathrm{~nm}$, mirroring the negative region of DAS2, thus describing a delayed onset of the latter. The intermediate component (DAS3, grey line) describes
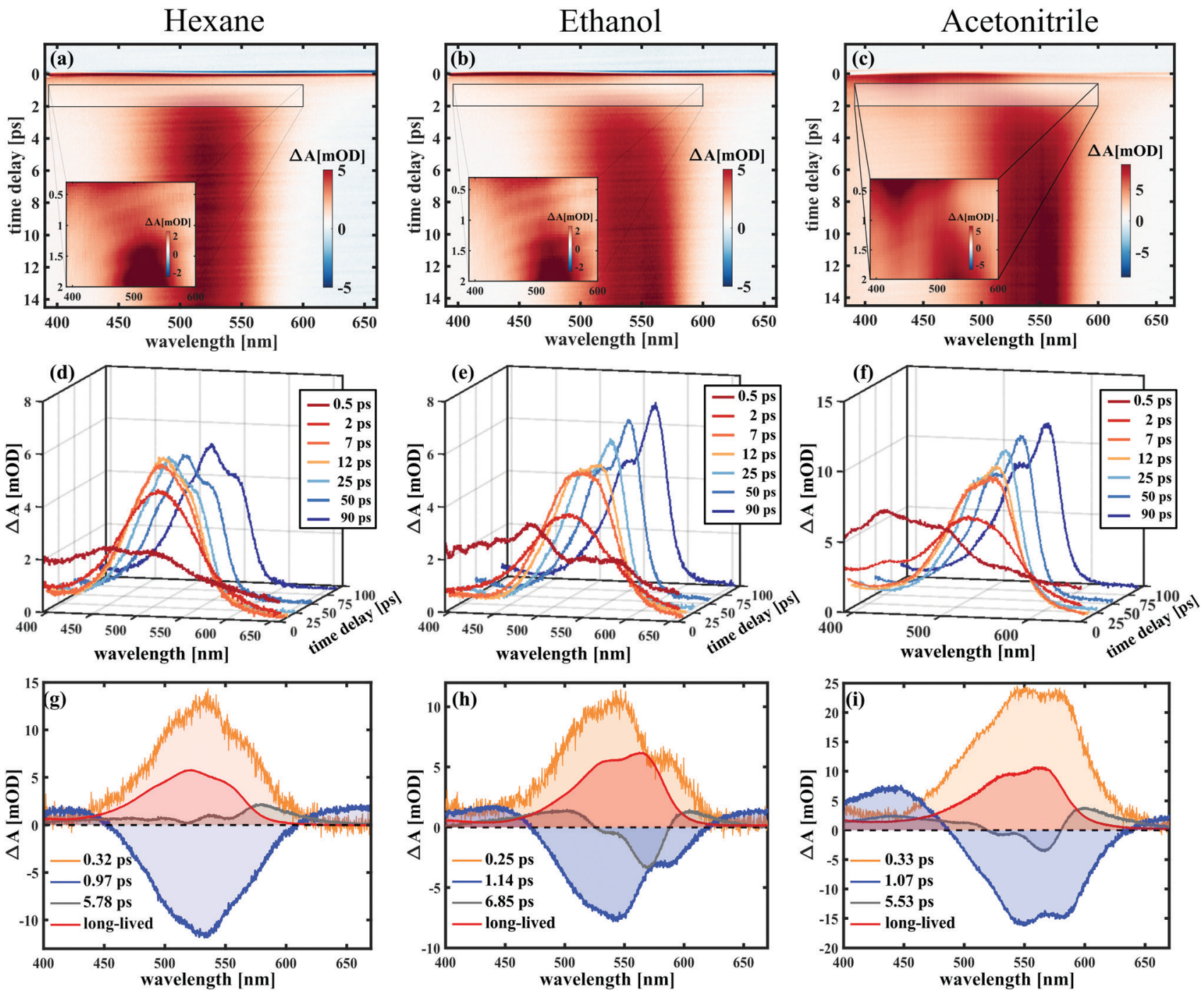

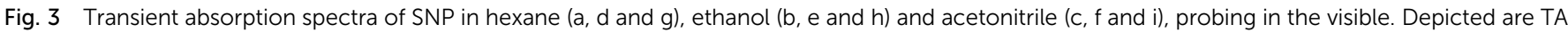

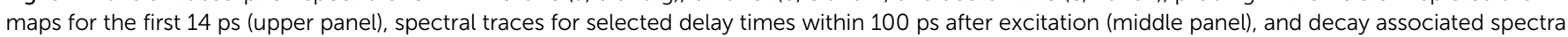
from global fitting (lower panel) for all three solvents. 

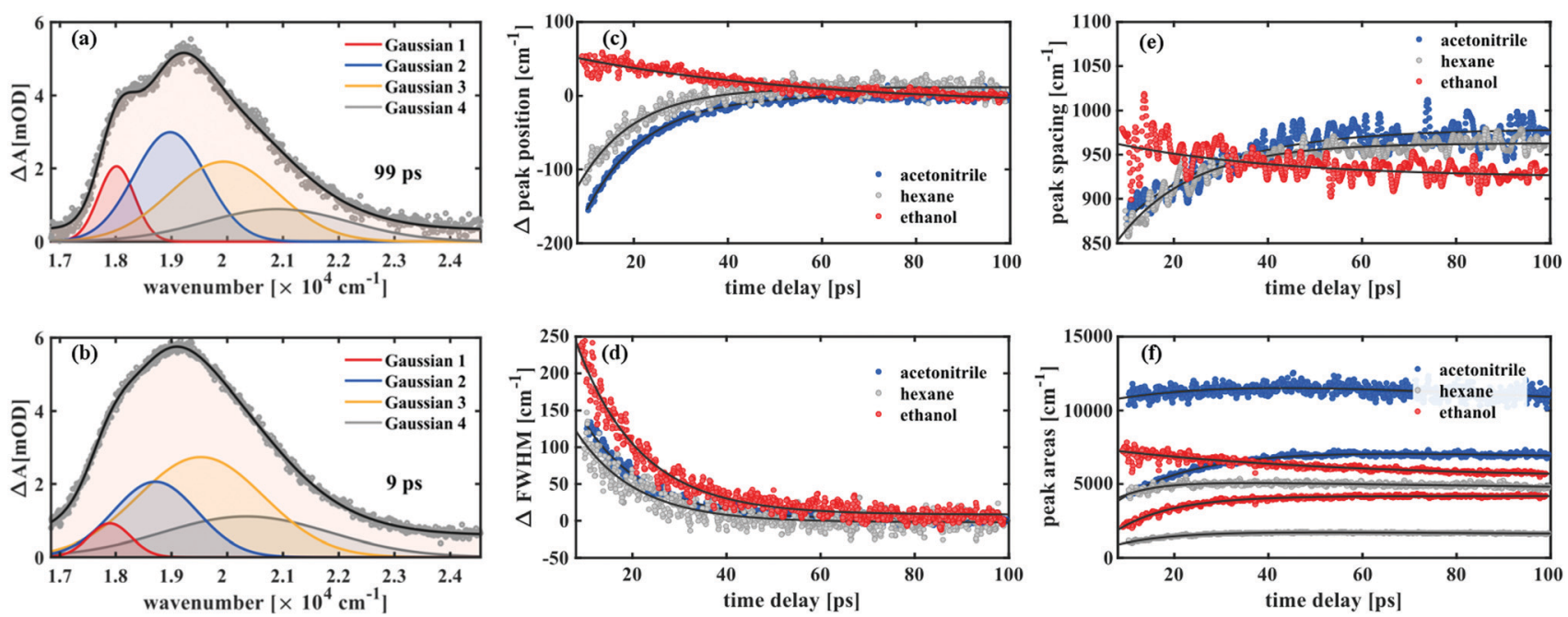

Fig. 4 Line shape analysis of the transient UV/vis absorption data. The Gaussian components are labeled from 1 to 4 , where Gaussian 1 refers to the peak at the lowest energy and Gaussian 4 is the highest energy peak. The fit is shown for the spectra in hexane at 99 ps (a) and 9 ps (b) after excitation representing the analysed time window. The changes in peak position and FWHM values of Gaussian 1 for all three solvents are shown in (c) and (d), respectively. (e) Shows the peak spacings and (f) the areas of the first two Gaussians for each solvent (in all three cases, the larger area belongs to the second peak).

the spectral development from the initial band towards the longlived component (DAS4, red line), which represents the spectrum at 14 ps. Although the global fitting represents the data well, interpreting the time-dependent spectral shift is not straightforward. Therefore, we treat the intermediate time constant in a separate analysis involving decomposition of the spectra into Gaussian functions, which is described in the following.

Such a dynamic Gaussian line shape analysis is able to describe spectral shifts and provides information about the temporal evolution of the peaks constituting the spectra. For this purpose, the spectra (converted to wavenumbers) for each time point were fit with a sum of four Gaussian functions (three in case of ethanol) tracking their respective spectral positions, widths and areas from $100 \mathrm{ps}$ down to $9 \mathrm{ps}$ after excitation. A detailed description of the fitting procedure and a model comparison can be found in the ESI. $\dagger$ After careful evaluation of different constraints to the parameters it was found that the data in this time window could be described properly by a simplified model, which ascribes the spectral fine structure to a vibrational progression with equal spacings between the peaks and a linear increase in peak width from lowest transition energy to highest. It was thus possible to fit all peak widths $w_{i}$ with a single parameter $w_{1}$ by using the relation $w_{i}=i \cdot w_{1}$ with $i$ being the peak number. The peak positions were characterised by one parameter for the absolute position $b_{1}$ and one for the peak spacing $\delta$ related by $b_{i}=b_{1}+(i-1) \cdot \delta$. All three parameters exhibited a monoexponential behaviour over time and the respective time constants are shown in Table 1.

Notably, both in acetonitrile and hexane, a time constant of 13 ps was found for the relative changes in peak positions, which blue-shift, and for the peak widths, which decay over time. While in ethanol a similar decay constant (14 ps) was obtained for the FWHM values, the aforementioned red-shift is reflected in the peak position changes with a time constant of
Table 1 Time constants from fits of the evolution of position $\left(\tau_{\text {pos }}\right.$ for overall peak position and $\tau_{\mathrm{spac}}$ for the peak spacing) and FWHMs $\left(\tau_{\mathrm{FWHM}}\right)$ of the Gaussian peaks (key: $r$ - rise; $d$ - decay)

\begin{tabular}{llll}
\hline & $\tau_{\text {pos }}[\mathrm{ps}]$ & $\tau_{\text {spac }}[\mathrm{ps}]$ & $\tau_{\text {FWHM }}[\mathrm{ps}]$ \\
\hline Acetonitrile & $13 \pm 0.2(\mathrm{r})$ & $21 \pm 3(\mathrm{r})$ & $13 \pm 0.3(\mathrm{~d})$ \\
Hexane & $13 \pm 0.5(\mathrm{r})$ & $16 \pm 1(\mathrm{r})$ & $13 \pm 0.8(\mathrm{~d})$ \\
Ethanol & $50 \pm 5(\mathrm{~d})$ & $33 \pm 8(\mathrm{~d})$ & $14 \pm 0.4(\mathrm{~d})$
\end{tabular}

$50 \mathrm{ps}$. Furthermore, in the former two solvents the peak spacing increases over time, while decreasing with a time constant of $35 \mathrm{ps}$ in case of ethanol.

3.2.2 UV-UV. The transient UV absorption map of SNP in $n$-hexane is shown in Fig. 5 . Measurements were also carried out in ethanol but exhibited no qualitative difference (see ESI $\dagger$ ). Around time zero, following the CPM signal, an intense absorption covering the entire measured range is observed. As the initial absorption decays within 2 ps, a negative feature between 270 and $305 \mathrm{~nm}$ and a positive feature between 305 and $340 \mathrm{~nm}$ start to dominate. After this time, the spectra do not change significantly either in their intensities or profiles within the measured time window up to one nanosecond (see Fig. 5d). The data were fitted using two decay associated spectra, one of which gave a time constant of $0.6-0.7$ ps representing the broad absorption near time zero, whereas the second time constant exceeded the measurement time window ( $\gg 1 \mathrm{~ns}$ ).

\subsection{Excited-state dynamics simulations}

The populations of the first four electronic states over time obtained from semi-classical excited state dynamics simulations and averaged over all 30 trajectories are depicted in Fig. 6. Starting with an initial population of 0.5 in both the $\mathrm{S}_{2}$ and the $\mathrm{S}_{3}$ state, the dynamics proceed via internal conversion processes into $S_{1}$. Subsequently, the molecules relax to the ground state of 

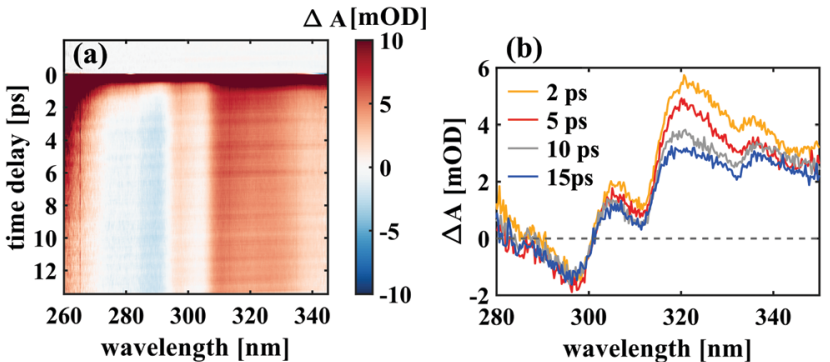

(c)
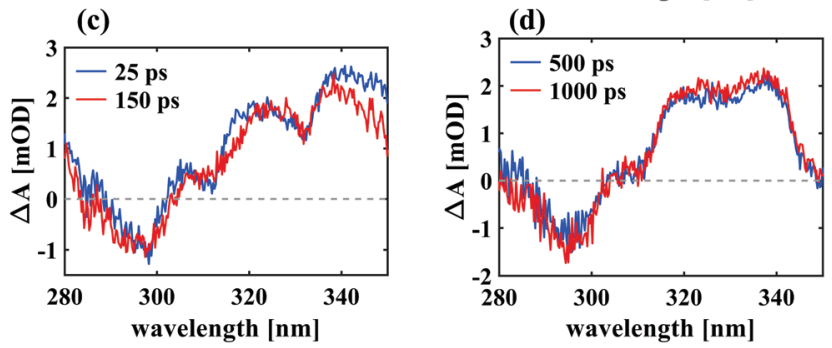

Fig. 5 UV transient absorption of SNP in hexane, excited at $266 \mathrm{~nm}$. An intense broad absorption around time zero decays within 1 ps revealing a long-lived difference spectrum with decreased absorption between 270 and $305 \mathrm{~nm}$ and increased absorption between 305 and $340 \mathrm{~nm}$ with respect to the closed-ring form.

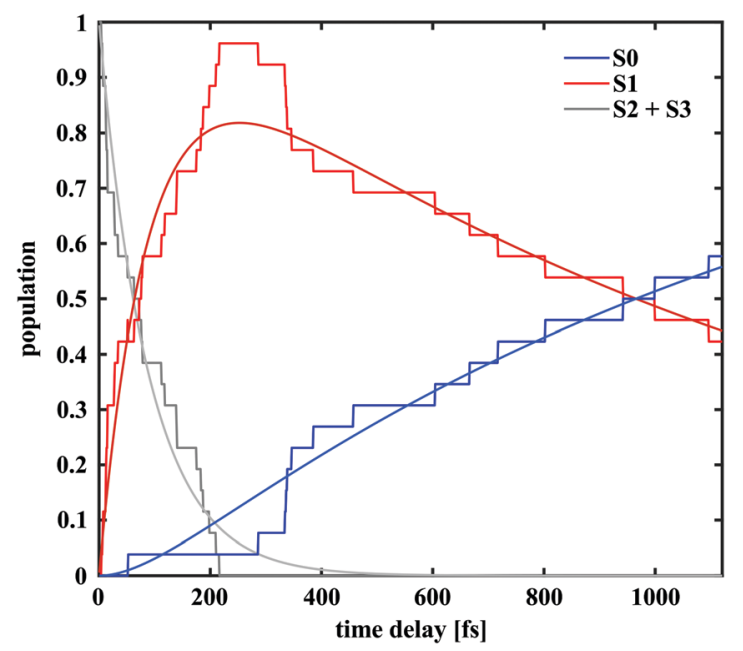

Fig. 6 Populations of the first four electronic states of SNP after UV excitation according to the excited state dynamics simulation. The data were averaged over 30 trajectories and fit with a kinetic model.

either the merocyanine or the closed-ring form. The populations were fitted with a unidirectional, sequential kinetic model to obtain the transfer rates between states. To enable an accurate fitting of the $S_{1}$ population dynamics, the decay of the initially occupied superposition state between $S_{3}$ and $S_{2}$ was described with a single rate constant. The resulting time constant for the transfer to $S_{1}$ was $90 \mathrm{fs}$, while transfer from $S_{1}$ to $S_{0}$ occurred with a time constant of 1.3 ps. This rate constant represents a combination of all relaxation pathways to the ground states of either the SNP or the MC form.

In 14 of the 30 simulated trajectories, $\mathrm{C}-\mathrm{O}$ bond breaking occurred along the $\mathrm{S}_{1}$ vibrational relaxation coordinate and the carbon hybridisation changed from $\mathrm{sp}^{3}$ to $\mathrm{sp}^{2}$. As a result of this, the two orthogonal moieties rotated with respect to each other along the methine bridge. This bridge can be described by using the three dihedral angles $\alpha, \beta$ and $\gamma$ along it (see Fig. 1). The various orientations of these angles determine the final conformer to be formed. Notably, only one of these trajectories led back to the spiro form, once the bond had been broken.

Plots of the $\mathrm{C}-\mathrm{O}$ bond distances during the first 700 fs for both trajectories yielding the open-ring form and trajectories leading back to the closed form are shown in Fig. 7. The bond breaking trajectories involve rapid elongation of the $\mathrm{C}-\mathrm{O}$ bond from the equilibrium distance of $1.5 \AA$ to about $3.5 \AA$ A Subsequently, the $\mathrm{C}-\mathrm{O}$ distances fluctuate around that value and any further changes upon conversion towards the planar form occur on a longer time scale. To characterise the ring-opening time, the formation of open-ring isomers was obtained using a $\mathrm{C}-\mathrm{O}$ bond elongation threshold of $1 \AA$ and fitted with an exponential function starting at $90 \mathrm{fs}$. This yielded a ring-opening time constant of $350 \mathrm{fs}$ (see ESI $\dagger$ ). Based on the geometry optimisation on the first excited state, a minimum on the $S_{1}$ potential energy surface was found corresponding to an open-ring non-planar structure with a C-O distance of $2.7 \AA$ and dihedral angles of $\alpha=-132^{\circ}, \beta=5.9^{\circ}$ and $\gamma=43.8^{\circ}$.

In case of the trajectories where the molecules did not undergo $\mathrm{C}-\mathrm{O}$ bond breaking but relaxed to the ground state of SNP, a temporary elongation of the bond between the indoline nitrogen and the spiro-carbon of $1.1 \pm 0.1 \AA$ for a duration of $100 \pm 30$ fs occurred during the transfer from $S_{1}$ to $S_{0}$. The temporal correlation between the elongation and relaxation to $S_{0}$ is demonstrated in Fig. 8 and is in agreement with previously reported CASPT2/CASSCF studies on BIPS. ${ }^{28}$

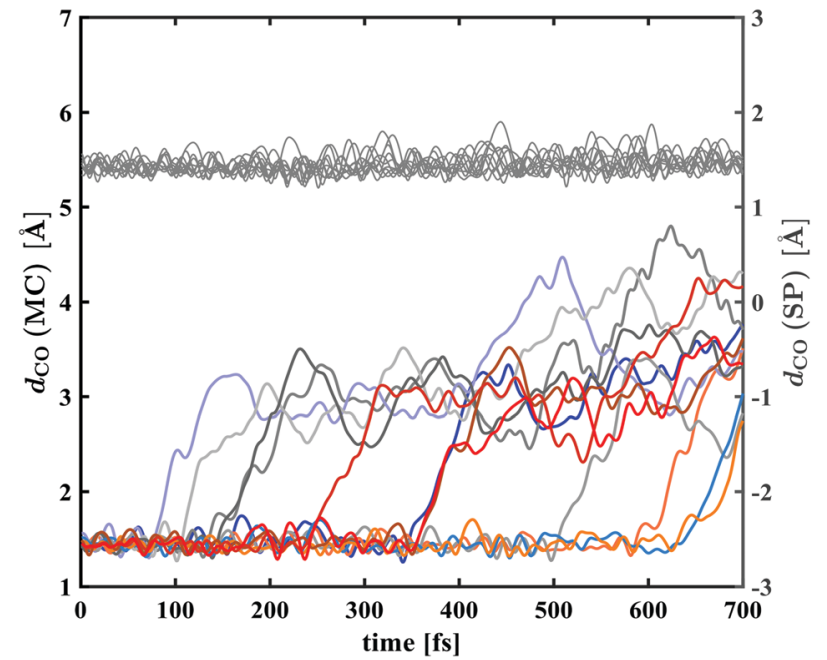

Fig. 7 Bond distance $d_{\mathrm{CO}}$ between the spiro-carbon and the pyran oxygen for the excited state dynamics trajectories leading to MC (coloured lines, left ordinate) and trajectories leading back to the SNP ground state (grey lines, right ordinate). In the $\mathrm{MC}$ trajectories, $\mathrm{C}_{\text {spiro }}-\mathrm{O}$ bond breaking occurs via rapid elongation of the bond of about $1.5 \AA$ taking less than 100 fs and no return to a closed-ring isomer was found. In contrast, no significant changes in $\mathrm{C}_{\text {spiro }}-\mathrm{O}$ bond length were present in the trajectories leading back to the ground state of SNP. 


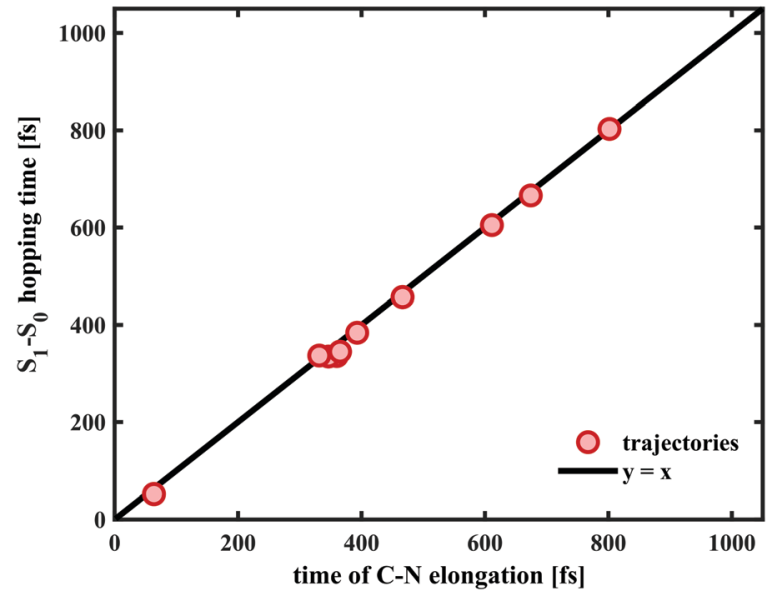

Fig. 8 Temporal correlation between the transfer from $S_{1}$ to $S_{0}$ and the $\mathrm{C}_{\text {spiro }}-\mathrm{N}$ elongation of $1.1 \pm 0.1 \AA$ in the trajectories that did not undergo $\mathrm{C}_{\text {spiro }}-\mathrm{O}$ bond breaking. The values on the abscissa for each trajectory were obtained by taking the mean of the time points during which the $\mathrm{C}_{\text {spiro }}-\mathrm{N}$ bond was elongated above the half-maximum value. The black line $(y=x)$ is shown as reference for a perfect correlation.

\subsection{Discussions}

3.4.1 Ring-opening and isomerisation dynamics. A schematic energy diagram of the ring-opening dynamics of SNP based on our interpretation of the experimental and theoretical results is presented in Fig. 9. Upon $266 \mathrm{~nm}$ excitation, the ground state of SNP is depopulated and high-lying excited states are populated. According to our simulations, internal conversion from $\mathrm{S}_{3}$ and $\mathrm{S}_{2}$ to the $S_{1}$ state occurs rapidly with a time constant of $90 \mathrm{fs}$, which is below our experimental time resolution. The instrument response limited, positive signal observed in the TA experiments (Fig. 3) can thus be attributed to absorption from the $S_{1}$ state, in agreement with previously reported assignments. ${ }^{16,17}$

On the pathway leading to the MC product (see Fig. 9a), the wave packet propagates on the $S_{1}$ potential energy surface towards an excited state minimum. During this propagation, the bond between the spiro-carbon and the pyran oxygen is elongated and eventually broken. This results in an open-ring intermediate with nearly orthogonal arrangement of the two subunits according to the $S_{1}$ geometry optimisation. The formation of this intermediate manifests as a delayed onset of the product band, which is found to be around $300 \mathrm{fs}$ (see Section 3.2.1) and is in good agreement with the bond breaking time of 350 fs obtained from simulations. Importantly, our simulations show that the ring-opening dynamics occur exclusively on the $S_{1}$ surface regardless of the excitation wavelength. Subsequent propagation in the next 0.8-0.9 ps involves crossing of an energy barrier on the $S_{1}$ surface and the passage through a conical intersection (CI) towards the planar MC configuration in the electronic ground state. This interpretation is consistent with the observed red-shift of $\sim 100 \mathrm{~nm}\left(4000 \mathrm{~cm}^{-1}\right)$ in the absorption spectrum occurring between 0.3 and $1.1 \mathrm{ps}$. The fact that all subsequently observed spectral shifts are either towards shorter wavelengths or at least an order of magnitude smaller supports the suggestion that no further planarisation takes place.

As our simulations show that only one out of 14 trajectories involving breaking of the $\mathrm{C}-\mathrm{O}$ bond led back to the spiro form, we conclude that the bifurcation into two main relaxation channels occurs earlier than 300 fs post-excitation. The key driving mode for internal conversion to the spiro form (see Fig. 9b) has been proposed to involve $\mathrm{C}-\mathrm{N}$ elongation in a theoretical study
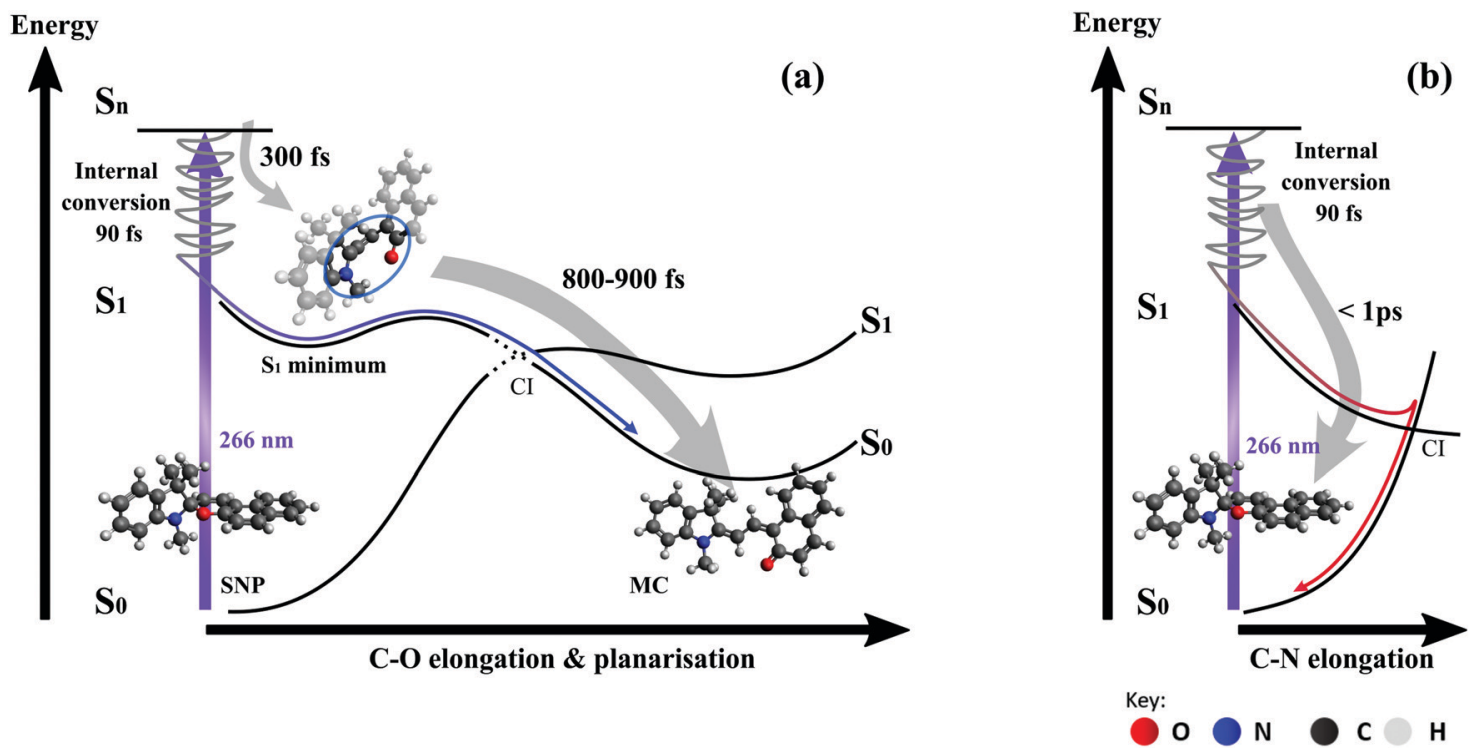

Fig. 9 Schematic illustration of the reaction pathways along the relevant reaction coordinates. Upon excitation at $266 \mathrm{~nm}$, internal conversion to the $\mathrm{S}_{1}$ potential energy surface (PES) occurs within 90 fs according to simulations. (a) On the trajectory leading to $M C$, the $C_{\text {spiro }}-O$ bond is broken leading to an open-ring intermediate with a formation time of $300 \mathrm{fs}$ according to experiments (350 fs in simulations). The $S_{1}$ minimum structure shown here was found by geometry optimisation. The subsequent planarisation and relaxation to the ground state of MC occurs with a time constant between 800 and 900 fs based on our experimental data. (b) Relaxation back to the ground state of the closed-ring form involves elongation of the $\mathrm{C}_{\text {spiro }}-\mathrm{N}$ bond rather than the $\mathrm{C}_{\text {spiro }}-\mathrm{O}$ bond, according to our simulations and previously reported PES calculations. Transient absorption measurements in the UV range indicate that this relaxation takes less than 1 ps. 
mapping the potential energy surfaces ${ }^{28}$ which is also corroborated from our excited-state dynamics simulations.

Important insight regarding the time scales of both reaction pathways can be gained from the transient UV absorption data (see Fig. 5). The long-lived differential absorption can be attributed to depletion of the spiro-form and formation of MC and both positive and negative signals in this range do not change significantly after 1-2 ps, except for residual decay of the strong excited state absorption. Besides further supporting our previous assignment of the MC formation time, this observation suggests that relaxation back to SNP also occurs within 1 ps or less.

3.4.2 Merocyanine ground-state dynamics and solvent effects. Based on the interpretations given in the previous section, we assign the spectral dynamics observed after 2 ps in the visible range mainly to relaxation of the planar open-ring isomer in the electronic ground state. This is discussed in the following based on the outcome of our line shape analysis (Fig. 4).

In all cases, a narrowing of the bands was found with a time constant of $13 \mathrm{ps}$ for acetonitrile and hexane and $14 \mathrm{ps}$ for ethanol. The reason for narrowing can be that the product configuration space is converging towards the most stable structural conformer ${ }^{37}$ or dissipation of vibrational energy to the environment. ${ }^{38,39}$ The observed overall blue-shift of the product band in hexane and acetonitrile can be explained analogously in the context of change in occupation number of vibrational states from higher to lower due to intramolecular vibrational relaxation in the electronic ground state and vibrational cooling via collisions with surrounding solvent molecules. The excellent match of the time scales between the blue-shift and the narrowing in these two solvents supports this assignment.

The latter effect is masked in case of ethanol by a dynamic decrease in transition energies resulting in a red-shift of $50 \mathrm{~cm}^{-1}$ within 10 and 100 ps. Furthermore, the energy difference between the vibrational levels decreases over time in ethanol in contrast to the other two solvents. We note that out of the three solvents used in this study, ethanol is the only one capable of forming hydrogen bonds with merocyanine. In addition, its viscosity is higher by a factor of 3 compared to the other two solvents $\left(1.1 \times 10^{-3} \mathrm{~Pa}\right.$ s for ethanol and $\sim 0.3 \times 10^{-3} \mathrm{~Pa}$ s for both acetonitrile and $n$-hexane). These characteristics could account for the observed differences, but an unambiguous assignment exceeds the scope of this work. However, the particularity of solvation in ethanol has been reported previously and it has been shown by Horng et al., using time-resolved emission of coumarin 153 as solute, that ethanol exhibits significantly more complex relaxation dynamics than the other two solvents, with a long-lived component of $\sim 30 \mathrm{ps.}^{40} \mathrm{In}$ contrast, solvation times in acetonitrile $(\sim 0.6 \mathrm{ps})$ and non-polar solvents $(\sim 0.3 \mathrm{ps})$ were found to be in the sub-ps regime. ${ }^{40,41}$ This suggests that in case of ethanol, the solvent relaxation causing a red-shift of the bands dominates the ps-dynamics whereas the effect would be invisible in cases of acetonitrile and hexane since the relaxation time scales coincide with those of the reaction itself. Thus, our observations and analyses reflect the distinctive solvent relaxation dynamics in ethanol.

An unambiguous interpretation of the peak area dynamics is not possible without additional information about the excited state PES and the corresponding vibrational wavefunctions and Franck-Condon factors. The possibility of interconversion between different MC conformers further complicates the interpretation. The fact that the spectra could be adequately described by a simple vibrational progression model implies that the peaks in the final spectrum correspond to a vibrational fine structure originating from a single conformer. The energy difference between the peaks is found to be on the order of $1000 \mathrm{~cm}^{-1}$, which falls well within the stretching frequency of $\mathrm{C}-\mathrm{C}$ bonds in organic molecules. It should be stressed however that the identity of the conformer that is formed cannot be revealed with certainty by means of electronic spectroscopy, since the merocyanine isomers are geometrically close and likely to have similar electronic structures. This has recently been demonstrated by Xiong et al. for the closely related spironaphthoxazine. ${ }^{7}$

The key insight from this analysis of the spectral dynamics between 9 and 100 ps is thus, that they can be ascribed purely to thermalisation (13 ps) of the product and in case of ethanol solvent equilibration $(\sim 50 \mathrm{ps})$. This confirms that the chemical reaction itself takes place on a faster time scale.

\section{Conclusion}

Combining semi-classical nuclear dynamics simulations and transient absorption spectroscopy in both the visible and the UV, the present study provides deeper insight into the mechanism and following product equilibration of the photochemical reaction of unsubstituted spiropyrans. Thorough analysis of the spectral data up to 100 ps after excitation allowed differentiating between the dynamics of the isomerisation reaction itself and product relaxation and equilibration. Evidence was found for an open-ring reaction intermediate being formed within $300 \mathrm{fs}$ which subsequently planarises yielding the merocyanine form in a vibrationally hot ground state within $1.1 \mathrm{ps}$. Theoretical calculations confirm the existence of such an intermediate with an open-ring, non-planar geometry. According to the dynamics simulations, all trajectories that showed $\mathrm{C}-\mathrm{O}$ bond breaking led to the planar $\mathrm{MC}$, whereas trajectories returning to the SNP ground state did not exhibit any significant $\mathrm{C}-\mathrm{O}$ bond elongation, but were driven by a $\mathrm{C}-\mathrm{N}$ stretch mode in accordance with previously reported calculations. The internal conversion back to the initial state was found to occur within less than $1 \mathrm{ps}$.

The product relaxation dynamics could be described with a simplified vibrational progression model suggesting that vibrational relaxation takes places with a time constant of $\sim 13 \mathrm{ps}$. While the chemical dynamics did not exhibit any considerable solvent dependence, product equilibration in ethanol was influenced by solvent relaxation dynamics. With the present study, we have demonstrated that a dynamic line shape analysis is a useful method to describe time-dependent spectral shifts and spectral fine structure, complementing conventional global fit routines.

\section{Author contributions}

S. F. B., R. D. and K. M. S. contributed equally to this work. K. M. S., G. C. and S. F. B. constructed the experimental setup. S. F. B. and 
K. M. S. led the experimental front. Data analysis was carried out by S. F. B., K. M. S., R. D., S. A. H. and V. I. P. R. D. led the theoretical efforts with contributions from M. K. A. R., S. F. B. and K. M. S. prepared samples for the experiments. All authors contributed towards data interpretations and discussions. The project was conceived by R. J. D. M. and directed by R. J. D. M. and K. M. S.

\section{Conflicts of interest}

The authors declare no conflicts of interest.

\section{Acknowledgements}

This work was funded by the Max Planck Society. We thank Dr Hong Guang-Duan and Dr Ajay Jha for scientific advice and Dr Arunangshu Debnath and Dr Zheng Li for helpful discussions. This work made use of the GWDG High Performance Computing Facility at Göttingen (https://www.gwdg.de/applicationservices/high-performance-computing). Open Access funding provided by the Max Planck Society.

\section{References}

1 Photochromism: Molecules and Systems, ed. H. Dürr and H. Bouas-Laurent, Elsevier BV, 2003.

2 M. El Gemayel, K. Börjesson, M. Herder, D. T. Duong, J. Hutchison, C. Ruzié, G. Schweicher, A. Salleo, Y. Geerts, S. Hecht, E. Orgiu and P. Samorì, Nat. Commun., 2015, 6, 5054-5075.

3 E. Orgiu, N. Crivillers, M. Herder, L. Grubert, M. Pätzel, J. Frisch, E. Pavlica, D. T. Duong, G. Bratina, A. Salleo, N. Koch, S. Hecht and P. Samorì, Nat. Chem., 2012, 4, 675-679.

4 D. Bléger and S. Hecht, Angew. Chem., Int. Ed., 2015, 54, 11338-11349.

5 G. Berkovic, V. Krongauz and V. Weiss, Chem. Rev., 2000, 100, 1741-1754.

6 M. Irie, T. Fukaminato, K. Matsuda and S. Kobatake, Chem. Rev., 2014, 114, 12174-12277.

7 Y. Xiong, A. V. Jentzsch, J. W. M. Osterrieth, E. Sezgin, I. V. Sazanovich, K. Reglinski, S. Galiani, A. W. Parker, C. Eggeling and H. L. Anderson, Chem. Sci., 2018, 9, 3029-3040.

8 C. Beyer and H.-A. Wagenknecht, J. Org. Chem., 2010, 75, 2752-2755.

9 M. Livendahl, J. Jamroskovic, M. Hedenström, T. Görlich, N. Sabouri and E. Chorell, Org. Biomol. Chem., 2017, 15, 3265-3275.

10 R. Klajn, Chem. Soc. Rev., 2014, 43, 148-184.

11 J. Buback, P. Nuernberger, M. Kullmann, F. Langhojer, R. Schmidt, F. Würthner and T. Brixner, J. Phys. Chem. A, 2011, 115, 3924-3935.

12 H. Takahashi, K. Yoda, H. Isaka, T. Ohzeki and Y. Sakaino, Chem. Phys. Lett., 1987, 140, 90-94.

13 E. Fischer and Y. Hirshberg, J. Chem. Soc., 1952, 4522-4524.

14 R. Heiligman-Rim, Y. Hirshberg and E. Fischer, J. Chem. Soc., 1961, 156-163.
15 A. Kellmann, F. Tfibel, R. Dubest, P. Levoir, J. Aubard, E. Pottier and R. Guglielmetti, J. Photochem. Photobiol., A, 1989, 49, 63-73.

16 N. P. Ernsting, B. Dick and T. Arthen-Engeland, Pure Appl. Chem., 1990, 62, 1483-1488.

17 N. P. Ernsting and T. Arthen-Engeland, J. Phys. Chem., 1991, 95, 5502-5509.

18 N. P. Ernsting, Chem. Phys. Lett., 1989, 159, 526-531.

19 M. Rini, A.-K. Holm, E. T. J. Nibbering and H. Fidder, J. Am. Chem. Soc., 2003, 125, 3028-3034.

20 J. Kohl-Landgraf, M. Braun, C. Özçoban, D. P. N. Gonçalves, A. Heckel and J. Wachtveitl, J. Am. Chem. Soc., 2012, 134, 14070-14077.

21 M. Suzuki, T. Asahi and H. Masuhara, Phys. Chem. Chem. Phys., 2002, 4, 185-192.

22 R. J. D. Miller, Science, 2014, 343, 1108-1116.

23 T. Elsaesser and W. Kaiser, Annu. Rev. Phys. Chem., 1991, 42, 83-107.

24 J. C. Owrutsky, D. Raftery and R. M. Hochstrasser, Annu. Rev. Phys. Chem., 1994, 45, 519-555.

25 G. A. Voth and R. M. Hochstrasser, J. Phys. Chem., 1996, 100, 13034-13049.

26 T. Brust, S. Malkmus, S. Draxler, S. A. Ahmed, K. RückBraun, W. Zinth and M. Braun, J. Photochem. Photobiol., A, 2009, 207, 209-216.

27 C. T. Middleton, B. Cohen and B. Kohler, J. Phys. Chem. A, 2007, 111, 10460-10467.

28 F. Liu and K. Morokuma, J. Am. Chem. Soc., 2013, 135, 10693-10702.

29 G. Zhai, S. Shao, S. Wu, Y. Lei and Y. Dou, Int. J. Photoenergy, 2014, 541791.

30 M. Frisch, G. Trucks, H. Schlegel, G. Scuseria, M. Robb, J. Cheeseman, G. Scalmani, V. Barone, B. Mennucci and G. Petersson et al., Gaussian 09, revision D. 01, 2009.

31 T. Yanai, D. P. Tew and N. C. Handy, Chem. Phys. Lett., 2004, 393, 51-57.

32 F. Weigend and R. Ahlrichs, Phys. Chem. Chem. Phys., 2005, 7, 3297-3305.

33 Principles of Fluorescence Spectroscopy, ed. J. R. Lakowicz, Springer US, 2006.

34 P. J. M. Johnson, V. I. Prokhorenko and R. J. D. Miller, Opt. Express, 2009, 17, 21488-21496.

35 V. I. Prokhorenko, A. Halpin, P. J. M. Johnson, R. J. D. Miller and L. S. Brown, J. Chem. Phys., 2011, 134, 085105.

36 I. H. van Stokkum, D. S. Larsen and R. van Grondelle, Biochim. Biophys. Acta, Bioenerg., 2004, 1657, 82-104.

37 G. Buntinx, O. Poizat, S. Foley, M. Sliwa, S. Aloïse, V. Lokshin and A. Samat, Dyes Pigm., 2011, 89, 305-312.

38 G. Buntinx, R. Naskrecki, C. Didierjean and O. Poizat, J. Phys. Chem. A, 1997, 101, 8768-8777.

39 G. Buntinx, R. Naskrecki and O. Poizat, J. Phys. Chem., 1996, 100, 19380-19388.

40 M. L. Horng, J. A. Gardecki, A. Papazyan and M. Maroncelli, J. Phys. Chem., 1995, 99, 17311-17337.

41 S. A. Kovalenko, J. L. Pérez Lustres, N. P. Ernsting and W. Rettig, J. Phys. Chem. A, 2003, 107, 10228-10232. 